\title{
Unsteady Finite Amplitude Convection of Water-Copper Nanoliquid in high-porosity Enclosures
}

\author{
P. G. Siddheshwar* \\ Professor \\ Department of Mathematics \\ Bangalore University \\ Bangalore 560056 India \\ Email: mathdrpgs@gmail.com \\ K. M. Lakshmi \\ Doctoral Student \\ Department of Mathematics \\ Bangalore University \\ Bangalore 560056 India \\ Email: lakshmikmmaths@gmail.com
}

\begin{abstract}
Unicellular Rayleigh-Bénard convection of water-copper nanoliquid confined in a high-porosity enclosure is studied analytically. The modified-Buongiorno-Brinkman two-phase model is used for nanoliquid description to include the effects of Brownian motion, thermophoresis, porous medium friction and thermophysical properties. Free-free and rigidrigid boundaries are considered for investigation of onset of convection and heat transport. Boundary effects on onset of convection are shown to be classical in nature. Stability boundaries in the $R_{1}^{*}-R_{2}$ plane are drawn to specify the regions in which various instabilities appear. Specifically, subcritical instabilities' region of appearance is highlighted. Square, shallow and tall porous enclosures are considered for study and it is found that the maximum heat transport occurs in the case of a tall enclosure and minimum in the case of a shallow enclosure. The analysis also reveals that the addition of a dilute concentration of nanoparticles in a liquidsaturated porous enclosure advances onset and thereby enhances the heat transport irrespective of the type of boundaries. The presence of porous medium serves the purpose of heat storage in the system because of its low thermal conductivity.
\end{abstract}

\section{Introduction}

Rayleigh-Bénard convection(RBC) of Newtonian liquid in the presence of suspended nanoparticles is termed as Rayleigh-Bénard convection of nanoliquids.

\footnotetext{
*Address all correspondence related to ASME style format and figures to this author.
}

Rayleigh-Bénard convection of nanoliquids is generally studied using one of the following models:

(i) Khanafer-Vafai-Lightstone single-phase model(KVLM) [1] or

(ii) Buongiorno two-phase model(BM) [2].

There are many reported works using the KVLM( [3], [4], [5], [6]). Siddheshwar and Meenakshi [3] analytically studied the RBC in nanoliquids by considering the thermophysical properties of 20 nanoliquids and they showed that among 20 nanoliquids, engine oil-silver nanoliquid transports maximum heat. Abu Nada [4] studied the effect of variable thermal conductivity and variable viscosity of $\mathrm{CuO}-$ water nanoliquid on heat transport and found that the Nusselt number is very sensitive to the viscosity models than the thermal conductivity models. Alloui et al. [5] studied both analytically and numerically the natural convection of nanoliquid confined in a rectangular shallow enclosure. They concluded that there is an optimum volume fraction, which is dependent on Rayleigh number, for each nanoparticles at which the heat transport is maximum. Alsabery et al. [6] numerically studied the natural convection in nanoliquid-saturated porous medium by considering four different nanoparticles and using the local thermal non-equilibrium(LTNE) model and they showed that LTNE model leads to enhanced heat transport compared to local thermal equilibrium(LTE) model. The drawback of the KVL model is that it can be thought of to be a byproduct of the corresponding problem involving a Newtonian fluid but with thermophysical properties being that of the nanoliquid. The results of this model can thus be recovered from the New- 
liquid properties and thereby with new definition of the classical parameters.

The Buongiorno model as used so far by many( [7], [8], [9], [10], [11], [12], [13], [14], [15], [16], [17], [18] [19], [20], [21]) deals with a nanoliquid as a binary fluid system with Soret effect and the model considers the thermophysical properties of the base liquid only. Tzou( [7], [8]) studied the RBC in nanoliquid and obtained the closed form solution for the Rayleigh number. Yang et al. [9] obtained exact solutions for a laminar nanofluid flow in channels and tubes. They achieved the maximum heat transport when the ratio of Brownian and thermophoretic diffusivities is nearly 0.5 . Sheikholeslami et al. [10] numerically investigated the natural convection in an enclosure filled with nanoliquid. They showed that the Lewis number has negligible effect on Nusselt number at smaller values of buoyancy ratio. Bhadauria and Agarwal [11] and Agarwal et al. [12] carried out linear and non-linear stability analyses in a nanoliquidsaturated porous medium by considering the LTNE model. They found that the effect of time on Nusselt number is oscillatory for small time scale and it reaches to steady value at long times. Nield and Kuznetsov [13] analytically studied the RBC in a nanoliquid-saturated porous medium using $\mathrm{BM}$ in both top heavy and bottom heavy arrangements of nanoparticles. They showed the possibility of oscillatory convection in bottom heavy distribution of nanoparticles. Kuznetsov and Nield [14] studied the linear stability analysis in a nanoliquid-saturated porous medium using the three-temperature model between porous matrix, base liquid and nanoparticles. They conclude that the effect of LTNE between porous matrix and nanoliquid is negligible in case of a dilute concentration of nanoparticles. Later in 2011, Kuznetsov and Nield [15] extended the study for Brinkman model. Khalili [16] experimentally investigated the nonhomogeneous distribution of nanoparticles in a nanoliquid using ANOVA method and they experimentally validated the assumption of non-homogeneous distribution of nanoparticles in the study. A linear stability analysis is performed by Yadav et al. [17] to find the effect of internal heat source on onset of convection with free-free, rigid-rigid and rigid-free boundary conditions. They found that the system is more stable in case of rigid-rigid boundary conditions. Analytical study of thermal instability in a finite depth of horizontal fluid layer is investigated by Agarwal and Rana [18]. Zero nanoparticle flux boundary condition was used in the study and they showed the impossibility of oscillatory convection for such boundary condition. Nield and Kuznetsov [19] analytically studied the onset of double diffusive convection in a nanoliquid and they obtained the results for both nonoscillatory and oscillatory cases. di Schio et al. [20] numerically studied the laminar forced convection in nanofluids using a Galerkin finite element method. They concluded that the concentration field will be strongly affected by the periodic boundary condition. A detailed discussion of literature survey and modelling of equations for forced, natural or mixed convection is given by Barletta et al. [21].

The serious drawback of the Buongiorno model was re- cently rectified by Siddheshwar et al. [22] who introduced thermophysical properties of the nanoliquid into the model. Phenomenological laws and mixture theory was used to model the thermophysical properties of the nanoliquid in terms of corresponding properties of base liquid and the nanoparticles. The BM with thermophysical properties of nanoliquid incorporated will henceforth be called modifiedBuongiorno model(MBM)( [23], [24], [25], [26], [27], [28], [29], [30]). A detailed information on the modelling of thermophysical properties of nanoliquids and numerical simulations of nanoliquids is discussed in the review article of Vanaki et al. [23]. Garoosi et al. [24] carried out the simulation for natural and mixed convection in nanoliquids occupying a differentially heated square enclosure. They concluded that there is an optimum value of a volume fraction for each combination of Rayleigh and Richardson numbers at which the heat transfer is at its peak. Haddad et al. [25] studied the presence and absence of Brownian motion and thermophoresis effects on heat transport in the RBC of a nanoliquid and they observed the enhanced heat transport even at a low volume fraction in the presence of the above two effects. Dastmalchi et al. [26] simulated natural convection using finite difference and control volume methods in a square porous enclosure filled with water- $\mathrm{Al}_{2} \mathrm{O}_{3}$ nanoliquid. Both homogeneous and non-homogeneous models were utilized in the study and they found that non-homogeneous model predicts a greater reduction of heat transfer in a system compared to homogeneous model. Siddheshwar and Kanchana( [27], [28]) studied RBC in twenty eight different nanoliquids confined in an enclosure bounded by stress-free boundaries. They showed that the additional modes in the solutions do not contribute much to the heat transport. Kanchana and Zhao [29] and Kanchana et al. [30] investigated the effect of internal heat generation on RBC in enclosures filled with nanoliquid. They compared the heat transport between the carbon nanotubes and alumina nanoparticles and they showed that the same quantity of heat transport can be achieved by replacing the carbon nanotubes with cheaper alumina nanoparticles but with an increased volume fraction.

There is no reported work on an analogous problem in porous media. The governing equations for a sparselypacked porous medium(proposed later on in the paper) shall be called modified-Buongiorno-Brinkman model(MBBM).

Rayleigh-Bénard convection with lateral boundary effects has been studied using the KVL model by several researchers( [31], [32], [33], [34], [35], [36], [37]). Park [31] derived the pseudo-single-phase model to study the RBC in a nanoliquid using the fact that the velocity and temperature of nanoparticles strictly follow those of the base liquid. $\mathrm{He}$ found the retortion of onset and reduction of heat transport in the presence of nanoparticles. Corcione [32] numerically investigated the RBC in nanoliquid. He developed two empirical models to find the effective thermal conductivity and dynamic viscosity of nanoliquids. He showed the existence of optimum particle loading for each nanoliquid at which the maximum heat transport is achieved. D'Orazio et al. [33] numerically investigated the RBC in tall rectangular enclosure using a SIMPLE-C algorithm. They found 
Copyright (that the flow pattern evolves from one-cell steady $\rightarrow$ two-cell steady $\rightarrow$ two-cell steady $\rightarrow$ two-cell periodic $\rightarrow$ one to threecell periodic $\rightarrow$ three-cell periodic with increase in Rayleigh number. Abu Nada and Chamka [34] studied the natural convection heat transfer in a square enclosure filled with ethylene glycol-CuO nanoliqud with different variable thermal conductivity and viscosity models. They found that the behaviour of average Nusselt number was influenced more by viscosity models than by thermal conductivity models. Jou and Tzeng [35] investigated on heat transfer enhancement in a rectangular enclosure filled with a nanoliquid for various values of parameters like buoyancy parameter, aspect ratio and volume fraction. They found that the heat transfer coefficient varies directly with aspect ratio and volume fraction. They also developed an empirical relation between Nusselt number and volume fraction. RBC with three different nanoliquids is studied by Elhajjar et al. [36]. They considered a new modelling for specific heat and thermal expansion coefficient. They found that the addition of nanoparticles delays the onset of convection and reduces the heat transport. Similar result is observed by Abu-nada et al. [37] for $\mathrm{Al}_{2} \mathrm{O}_{3}$-water and $\mathrm{CuO}$-water nanoliquids by considering variable thermal conductivity and variable viscosity models. The reason for this thermodynamical incorrect results is the improper modelling of thermophysical properties(which is discussed in section 57. The corresponding problem using the BM has also been reported but most of them have the inherent short comings as outlined earlier. The counterpart of the problem in a porous enclosure has been investigated by Grosan et al. [38] and Sheremet et al. [39] using the KVL model. The corresponding work using the BM does not seem to have been investigated extensively so far(except Sheremet et al. [40]).

The objectives of the paper are to investigate the following unconsidered aspects in the thermoconvective problem of nanoliquids in a porous enclosure:

1. To present new equations to model conservation of linear momentum and energy for nanoliquid-saturated porous medium using BM for nanoliquid and LapwoodBrinkman equation for porous media. The equation of state is also modified suitably for the problem.

2. To include the thermophysical properties of nanoliquidsaturated porous medium using phenomenological laws and mixture theory.

3. To make linear and non-linear stability analyses considering two different boundary conditions (free-free, rigidrigid) on the upper and lower plates with the lateral walls assumed to be adiabatic and impermeable.

4. Showing the existence of subcritical motions in the system.

5. The stability of a fluid flow is dependent on whether the small superposed disturbance given to the system decays or grows with time. If the disturbances are non-decaying in the system then the principle of exchange of stability is said to hold good only when the disturbances are nonoscillatory with time.

Proving the validity of PES(Principle of Exchange of
Stabilities).

6. Recovering the results of KVL model from those of the MBB model.

7. Showing the connection between Lorenz model and Ginburg-Landau equation.

8. Demonstrating the boundedness of the solution of amplitude equation due to its cubic nonlinear term.

9. Identifying the enclosure that facilitates maximum transport of heat.

10. Proposing the problem as a heat storage/heat removal system.

\section{Mathematical Formulation}

\subsection{Problem Description}

We consider two-dimensional, unicellular RayleighBénard convection of a nanoliquid confined in a porous enclosure of height $\mathrm{h}$ and width $\mathrm{b}$ having isothermal, isoconcentration horizontal walls and adiabatic, impermeable vertical walls as shown in Fig. 11. We choose a left handed Cartesian co-ordinate system with $\mathrm{z}$ as the vertical axis.

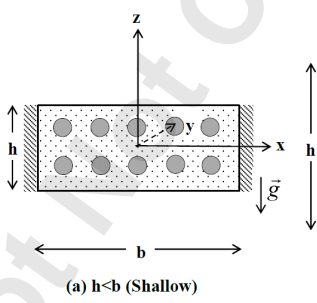

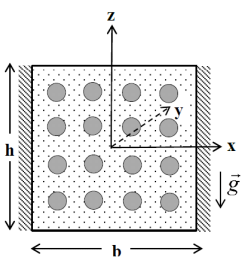

(b) $\mathbf{h}=\mathbf{b}$ (Square)

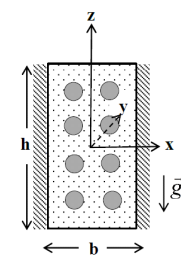

(c) $\mathbf{h}>\mathbf{b}$ (Tall)
Fig. 1: Schematic representation of nanoliquid-saturated high-porosity enclosures.

The modified-Buongiorno-Brinkman governing equations with the assumption of Boussinesq approximation are: Conservation of mass

$$
\nabla \cdot \vec{q}=0
$$

\section{Conservation of linear momentum}

$$
\begin{aligned}
& \rho_{0}\left[\frac{1}{\phi} \frac{\partial \vec{q}}{\partial t}+\frac{1}{\phi^{2}}(\vec{q} \cdot \nabla) \vec{q}\right]=-\nabla P+\mu \nabla^{2} \vec{q}-\frac{\mu_{n l}}{K} \vec{q} \\
& +\left[\rho_{0}-\rho_{0} \beta_{1}\left(T-T_{0}\right)+\rho_{0} \beta_{2}\left(C-C_{0}\right)\right] \vec{g},
\end{aligned}
$$

\section{Conservation of energy}

$$
M \frac{\partial T}{\partial t}+(\vec{q} \cdot \nabla) T=M \chi \nabla^{2} T
$$

The viscous dissipation term $\frac{\mu_{n l}}{\phi\left(\rho_{0} c\right)_{n l}}(\nabla \vec{q})^{2}$ has been neglected in the energy equation due to the assumption of Boussinesq approximation. Following the order of magni- 
tude analysis, it can be easily shown that $\frac{\mu_{n l}(\nabla \vec{q})^{2}}{\phi\left(\rho_{0} c\right)_{n l}(\vec{q} \cdot \nabla) T}=$ $\mathrm{o}\left(10^{-10}\right)$ (see appendix A).

\section{Conservation of nanoparticle concentration}

$$
\phi \frac{\partial C}{\partial t}+(\vec{q} \cdot \nabla) C=D_{B} \nabla^{2} C+\frac{D_{T}}{T_{0}} \nabla^{2} T
$$

where

$$
\begin{aligned}
& \nabla=\hat{i} \frac{\partial}{\partial x}+\hat{k} \frac{\partial}{\partial z}, D_{B}=\frac{\phi K_{B} T_{0}}{3 \pi \mu_{n l} d_{n p}}, D_{T}=\frac{0.26 \phi k_{n l}}{2 k_{n l}+k_{n p}} \frac{\mu_{n l} \alpha}{\rho_{0}}, \\
& \alpha=\frac{\text { volume of nanoparticles }}{\text { volume of (nanoparticles +base liquid) }}, 0 \leq \alpha<1, \\
& \phi=\frac{\text { volume of pores }}{\text { volume of (pores + porous material) }}, 0<\phi \leq 1,
\end{aligned}
$$

and other nanoliquid and nanoliquid-saturated porous medium properties are found from phenomenological laws or mixture theory:

\section{Phenomenological laws}

$$
\left.\begin{array}{rl}
\frac{\mu_{n l}}{\mu_{b l}} & =\frac{1}{(1-\alpha)^{2.5}}, \quad \frac{\mu}{\mu_{n l}}=\frac{1}{\phi^{2.5}}, \quad(\text { [41] }, \text { [42] }) \\
\frac{k_{n l}}{k_{b l}} & =\frac{\left(\frac{k_{n p}}{k_{b l}}+2\right)-2 \alpha\left(1-\frac{k_{n p}}{k_{b l}}\right)}{\left(\frac{k_{n p}}{k_{b l}}+2\right)+\alpha\left(1-\frac{k_{n p}}{k_{b l}}\right)}
\end{array}\right\}
$$

\section{Mixture theory}

$$
\begin{aligned}
& \rho_{0_{\mathrm{nl}}}=\alpha \rho_{0_{\mathrm{np}}}+(1-\alpha) \rho_{0_{\mathrm{bl}}}, \\
& \left(\rho_{0} c\right)_{\mathrm{nl}}=\alpha\left(\rho_{0} c\right)_{\mathrm{np}}+(1-\alpha)\left(\rho_{0} c\right)_{\mathrm{bl}}, c_{\mathrm{nl}}=\frac{\left(\rho_{0} c\right)_{\mathrm{nl}}}{\rho_{0_{\mathrm{nl}}}}, \\
& \left(\rho_{0} \beta_{1}\right)_{\mathrm{nl}}=\alpha\left(\rho_{0} \beta_{1}\right)_{\mathrm{np}}+(1-\alpha)\left(\rho_{0} \beta_{1}\right)_{\mathrm{bl}}, \beta_{1_{\mathrm{nl}}}=\frac{\left(\rho_{0} \beta_{1}\right)_{\mathrm{nl}}}{\rho_{0_{\mathrm{nl}}}}, \int^{(7)} \\
& \left.\left(\rho_{0} \beta_{2}\right)_{\mathrm{nl}}=\alpha\left(\rho_{0} \beta_{2}\right)_{\mathrm{np}}+(1-\alpha)\left(\rho_{0} \beta_{2}\right)_{\mathrm{bl}}, \beta_{2_{\mathrm{nl}}}=\frac{\left(\rho_{0} \beta_{2}\right)_{\mathrm{nl}}}{\rho_{0_{\mathrm{nl}}}} .\right) \\
& \rho_{0}=\phi \rho_{0_{\mathrm{nl}}}+(1-\phi) \rho_{0_{\mathrm{pm}}}, \\
& c=\frac{\phi\left(\rho_{0} c\right)_{\mathrm{nl}}+(1-\phi)\left(\rho_{0} c\right)_{\mathrm{pm}}}{\rho_{0}}, \\
& \beta_{1}=\frac{\phi\left(\rho_{0} \beta_{1}\right)_{\mathrm{nl}}+(1-\phi)\left(\rho_{0} \beta_{1}\right)_{\mathrm{pm}}}{\rho_{0}}, \\
& \beta_{2}=\frac{\phi\left(\rho_{0} \beta_{2}\right)_{\mathrm{nl}}+(1-\phi)\left(\rho_{0} \beta_{2}\right)_{\mathrm{pm}}}{\rho_{0}}, \\
& k=\phi k_{\mathrm{nl}}+(1-\phi) k_{\mathrm{pm}}, \quad k_{\mathrm{bl}_{\mathrm{eff}}}=\phi k_{\mathrm{bl}}+(1-\phi) k_{\mathrm{pm}}, \\
& \left.\chi=\frac{\phi k_{\mathrm{nl}}+(1-\phi) k_{\mathrm{pm}}}{\rho_{0} c}, M=\frac{\phi\left(\rho_{0} c\right)_{\mathrm{nl}}+(1-\phi)\left(\rho_{0} c\right)_{\mathrm{pm}}}{\left(\rho_{0} c\right)_{\mathrm{nl}}} .\right)
\end{aligned}
$$

In writing Eq. (2) we have considered linear Darcy friction only and neglected the Forchheimer(or Ergun) quadratic drag term which is important if we are considering large scale convective motions. On the assumption of small scale convective motion the inertial term(convective acceleration) also turns out to be negligible.

In modelling dynamic viscosity and thermal conductivity we have used the Brinkman model and Hamilton-Crosser model respectively. This is exactly the same modelling choice as that of modified Buongiorno model(MBM). Our choice of the MBB model in this theoretical investigation was made going by the choice of various other investigators considering Rayleigh-Bénard convection in nanoliquids. It must be pointed out here that an alternate, and possibly better, modelling of these two thermophysical properties can also be done using the Koo-Kleinstreuer model [44]. This shall, however, form the subject of an elaborate investigation in our future project.

Assuming the nanoliquid temperature and concentration of the nanoparticles to be constant at the boundaries, we may assume the boundary conditions on $\mathrm{T}$ and $\mathrm{C}$ to be:

$$
\begin{aligned}
& \left.\begin{array}{l}
T=T_{0}+\Delta T, C=C_{0}+\Delta C \text { at } z=\frac{-h}{2} \\
T=T_{0}, \quad C=C_{0} \quad \text { at } z=\frac{h}{2}
\end{array}\right\}, \frac{-b}{2}<x<\frac{b}{2}, \\
& \left.\frac{\partial T}{\partial x}=0, \frac{\partial C}{\partial x}=0 \text { at } x= \pm \frac{b}{2}\right\}, \frac{-h}{2}<z<\frac{h}{2}
\end{aligned}
$$

Two different boundary conditions on $\vec{q}$ will be considered on the perturbed quantity and shall be specified later.

\subsection{Basic State Solution}

At the basic state the temperature and concentration profiles are of conduction state and do not change with $\mathrm{t}$ and $\mathrm{x}$, and are given by

$$
\vec{q}=0, \quad P=P_{b}(z), \quad T=T_{b}(z), \quad C=C_{b}(z)
$$

Eq. (11) satisfies Eq. (1) and Eqs. (2) - 4) in the basic state take the form:

$$
\begin{aligned}
& \frac{d P_{b}}{d z}+\left[\rho_{0}-\left(\rho_{0} \beta_{1}\right)\left(T_{b}-T_{0}\right)+\left(\rho_{0} \beta_{2}\right)\left(C_{b}-C_{0}\right)\right] g=0, \\
& \frac{d^{2} T_{b}}{d z^{2}}=0, \frac{d^{2} C_{b}}{d z^{2}}=0 .
\end{aligned}
$$

On solving Eqs. (12), subject to the boundary conditions (9), we get

$$
T_{b}(z)=T_{0}+\left(\frac{1}{2}-\frac{z}{h}\right) \Delta T, C_{b}(z)=C_{0}+\left(\frac{1}{2}-\frac{z}{h}\right) \Delta C
$$

The expression of the basic state pressure distribution is not required in the analysis further on and hence is not recorded here. 
We now superimpose a perturbation on the quiescent basic state as given below:

$$
\vec{q}=\vec{q}^{\prime}, \quad P=P_{b}+P^{\prime}, \quad T=T_{b}+T^{\prime}, \quad C=C_{b}+C^{\prime}
$$

where prime denotes perturbed quantity.

Substituting Eq. (14) in the governing equations (1)-(4), using the basic state Eqs. (12), eliminating pressure from the resulting equations and introducing the stream function in the form $u=\frac{\partial \psi}{\partial z}, \quad w=-\frac{\partial \psi}{\partial x}$, we get the following three equations governing the perturbation:

$$
\begin{aligned}
& \frac{\rho_{0}}{\phi} \frac{\partial}{\partial t}\left(\nabla^{2} \psi\right)=\mu \nabla^{4} \psi-\frac{\mu_{n l}}{K} \nabla^{2} \psi \\
& -\left[\left(\rho_{0} \beta_{1}\right) \frac{\partial T^{\prime}}{\partial x}-\left(\rho_{0} \beta_{2}\right) \frac{\partial C^{\prime}}{\partial x}\right] g+\frac{\rho_{0}}{\phi^{2}} \frac{\partial\left(\psi, \nabla^{2} \psi\right)}{\partial(x, z)} \\
& M \frac{\partial T^{\prime}}{\partial t}=M \chi \nabla^{2} T^{\prime}-\frac{\Delta T}{h} \frac{\partial \psi}{\partial x}+\frac{\partial\left(\psi, T^{\prime}\right)}{\partial(x, z)} \\
& \phi \frac{\partial C^{\prime}}{\partial t}=D_{B} \nabla^{2} C^{\prime}+\frac{D_{T}}{T_{0}} \nabla^{2} T^{\prime}-\frac{\Delta C}{h} \frac{\partial \psi}{\partial x}+\frac{\partial\left(\psi, C^{\prime}\right)}{\partial(x, z)}
\end{aligned}
$$

\subsection{Non-dimensionalisation}

We non-dimensionalize Eqs. (15)-17) using the following dimensionless groups and we skip primes for simplicity:

$$
(X, Z)=\left(\frac{x}{b}, \frac{z}{h}\right), \Psi=\frac{\psi}{\chi_{b l}}, \Theta=\frac{T}{\Delta T}, \Phi=\frac{C}{\Delta C}, \tau=\frac{t \chi_{b l}}{h^{2}} .
$$

Using Eq. (18), the non-dimensional form of the governing Eqs. (15)-(17) are obtained in the form:

$$
\begin{gathered}
\frac{\Lambda}{\operatorname{Pr}} \frac{\partial}{\partial \tau}\left(\nabla_{A}^{2} \Psi\right)=\Lambda a \nabla_{A}^{4} \Psi-A^{2} \sigma^{2} a \nabla_{A}^{2} \Psi \\
+A^{4} a^{2}\left[R_{2} \frac{\partial \Phi}{\partial X}-R_{1} \frac{\partial \Theta}{\partial X}\right] \\
M \frac{\partial \Theta}{\partial \tau}=-A \frac{\partial \Psi}{\partial X}+a M \nabla_{A}^{2} \Theta+A \frac{\partial(\Psi, \Theta)}{\partial(X, Z)}, \\
\phi \frac{\partial \Phi}{\partial \tau}=-A \frac{\partial \Psi}{\partial X}+\frac{\varepsilon^{2} a N_{A}}{L e} \nabla_{A}^{2} \Theta+\frac{a}{L e} \nabla_{A}^{2} \Phi+A \frac{\partial(\Psi, \Phi)}{\partial(X, Z)},
\end{gathered}
$$

where

$$
R_{1}=\frac{\rho_{0} \beta_{1} \Delta T g b^{3}}{\mu_{n l} \chi}
$$

(Thermal Rayleigh number)

$R_{2}=\frac{\rho_{0} \beta_{2} \Delta C g b^{3}}{\mu_{n l} \chi}$,

(Concentration Rayleigh number)

$$
A=\frac{h}{b}
$$

(Aspect ratio)

$$
L e=\frac{\chi}{D_{B}},
$$

$\varepsilon^{2} N_{A}=\frac{D_{T} \Delta T}{D_{B} T_{0} \Delta C}$,

(modified diffusivity ratio)

$\operatorname{Pr}=\frac{\mu \phi}{\rho_{0} \chi}$,

(modified Prandtl number)

$a=\frac{\chi}{\chi_{b l}}$,

(Ratio of thermal diffusivities)

$$
\sigma^{2}=\frac{b^{2}}{K}
$$

(Porous parameter)

$$
\left.\Lambda=\frac{\mu}{\mu_{n l}},\right\} \text {. }
$$

(Brinkman number)

$\nabla_{A}^{2}=A^{2} \frac{\partial^{2}}{\partial X^{2}}+\frac{\partial^{2}}{\partial Z^{2}}$.

The Eqs. (19) to 21) are solved subject to two types of boundary conditions that are given below.

Case (i): Free-free, isothermal, iso-concentration horizontal boundaries and free-free, adiabatic, impermeable vertical boundaries

$$
\left.\begin{array}{r}
\Psi=\frac{\partial^{2} \Psi}{\partial Z^{2}}=\Theta=\Phi=0 \text { at } Z= \pm \frac{1}{2} ; X \in\left(\frac{-1}{2}, \frac{1}{2}\right), \\
\Psi=\frac{\partial^{2} \Psi}{\partial X^{2}}=\frac{\partial \Theta}{\partial X}=\frac{\partial \Phi}{\partial X}=0 \text { at } X= \pm \frac{1}{2} ; Z \in\left(\frac{-1}{2}, \frac{1}{2}\right) .
\end{array}\right\}
$$

Case (ii): Rigid-rigid, isothermal, iso-concentration horizontal boundaries and rigid-rigid, adiabatic, impermeable vertical boundaries

$$
\left.\begin{array}{c}
\Psi=\frac{\partial \Psi}{\partial Z}=\Theta=\Phi=0 \text { at } Z= \pm \frac{1}{2} ; X \in\left(\frac{-1}{2}, \frac{1}{2}\right), \\
\Psi=\frac{\partial \Psi}{\partial X}=\frac{\partial \Theta}{\partial X}=\frac{\partial \Phi}{\partial X}=0 \text { at } X= \pm \frac{1}{2} ; Z \in\left(\frac{-1}{2}, \frac{1}{2}\right) .
\end{array}\right\}
$$

Free-free boundary condition is an artificial and idealistic boundary condition but facilitates exact solution. However, rigid-rigid boundaries are realistic and will have to be dealt with approximately. Both Chandrasekhar even and odd functions satisfy the rigid boundary conditions. But the lowest characteristic value of critical Rayleigh number will occur for the even function. We choose the solution accordingly.

The details of a linear stability analysis is presented in section 5 in the context of diffusing stability boundaries. We now move on to perform a weakly nonlinear stability analysis of the system (19)-21) subject to boundary conditions (24) or 25).

\subsection{Weakly Non-linear Stability Analysis}

In a weakly non-linear stability analysis we require to describe the quantities $\Psi, \Theta$ and $\Phi$ in a non-linear convective regime. This can be done by considering the double Fourier series expansion for $\Psi, \Theta$ and $\Phi$. The effect of non-linearity comes into the study by the term $\sin (2 \pi Z)$ in $\Theta$, which represents the change in the horizontal mean by the distortion of the temperature field.

Case (i): The minimal mode representation of double 


$$
\left.\begin{array}{l}
\Psi=\sqrt{2} a M \delta^{2} /\left(A \pi^{2}\right) X_{1}(\tau) \cos (\pi X) \cos (\pi Z), \\
\Theta=-\sqrt{2} /\left(r_{1} \pi\right) Y_{1}(\tau) f_{1}(X, Z)-1 /\left(r_{1} \pi\right) Z_{1}(\tau) f_{2}(Z), \\
\Phi=-\sqrt{2} M /(\pi \phi) E_{1}(\tau) f_{1}(X, Z)-1 / \pi F_{1}(\tau) f_{2}(Z),
\end{array}\right\}
$$

where $f_{1}(X, Z)=\sin (\pi X) \cos (\pi Z), f_{2}(Z)=\sin (2 \pi Z)$ and $X_{1}, Y_{1}, Z_{1}, E_{1}, F_{1}$ are to be determined.

On substituting Eq. (26) into Eqs. (19)-21) and taking the orthogonality condition with the eigenfunctions, we get the following scaled Lorenz model:

$$
\begin{aligned}
U_{i} \frac{d X_{i}}{d \tau_{1}} & =a \operatorname{Pr}\left[Y_{i}-X_{i}-r_{\Phi_{i}} E_{i}\right] \\
\frac{d Y_{i}}{d \tau_{1}} & =a\left[r_{i} X_{i}-Y_{i}-X_{i} Z_{i}\right] \\
\frac{d Z_{i}}{d \tau_{1}} & =a\left[X_{i} Y_{i}-b^{*} Z_{i}\right] \\
\frac{d E_{i}}{d \tau_{1}} & =a\left[X_{i}-\frac{\varepsilon^{2} N_{A}}{L e} \frac{1}{r_{i} M} Y_{i}-\frac{1}{L e} \frac{1}{\phi} E_{i}-X_{i} F_{i}\right] \\
\frac{d F_{i}}{d \tau_{1}} & =a\left[-\frac{\varepsilon^{2} N_{A}}{L e} \frac{b^{*}}{r_{i} \phi} Z_{i}-\frac{1}{L e} \frac{b^{*}}{\phi} F_{i}+\frac{M^{2}}{\phi^{2}} X_{i} E_{i}\right]
\end{aligned}
$$

where $\mathrm{i}=1$ for case (i) and

$$
\left.\begin{array}{l}
\tau_{1}=\delta^{2} \tau, \quad U_{1}=\frac{M \delta^{6} \Lambda}{A^{5} \pi^{2}\left(R_{1_{c}}\right)_{1}}, \quad r_{\Phi_{1}}=\frac{R_{2}}{\left(R_{1_{c}}\right)_{1}} \frac{M}{\phi}, \\
r_{1}=\frac{R_{1}}{\left(R_{1_{c}}\right)_{1}},\left(R_{1_{c}}\right)_{1}=\frac{M \delta^{4}}{\pi^{2} A^{5}}\left(\Lambda \delta^{2}+A^{2} \sigma^{2}\right), b^{*}=\frac{4 \pi^{2}}{\delta^{2}} .
\end{array}\right\}
$$

Case (ii): The minimal mode representation of a double Fourier series for $\psi, \Theta, \Phi$ in this case is

$$
\left.\begin{array}{l}
\Psi=\frac{M a \delta^{2}}{2 \sqrt{2} A \pi \mu_{1} P_{5}} X_{2}(\tau) \mathrm{Cfe}_{x}(X) \mathrm{Cfe}_{z}(Z), \\
\Theta=\frac{-\sqrt{2} P_{4}}{r_{2} P_{5}} Y_{2}(\tau) f_{1}(X, Z)-\frac{P_{4}}{r_{2} P_{5}} Z_{2}(\tau) f_{2}(Z), \\
\Phi=\frac{-\sqrt{2} M P_{4}}{\phi P_{5}} E_{2}(\tau) f_{1}(X, Z)-\frac{P_{4}}{P_{5}} F_{2_{i}}(\tau) f_{2}(Z),
\end{array}\right\}
$$

where $\mathrm{Cfe}_{x}(X)$ and $\mathrm{Cfe}_{z}(Z)$ are the Chandrasekhar functions( [45], [46], [47]) given by

$$
\begin{aligned}
\mathrm{Cfe}_{x}(X) & =\frac{\cosh \left(\mu_{1} X\right)}{\cosh \left(\mu_{1} / 2\right)}-\frac{\cos \left(\mu_{1} X\right)}{\cos \left(\mu_{1} / 2\right)} \\
\mathrm{Cfe}_{z}(Z) & =\frac{\cosh \left(\mu_{1} Z\right)}{\cosh \left(\mu_{1} / 2\right)}-\frac{\cos \left(\mu_{1} Z\right)}{\cos \left(\mu_{1} / 2\right)}, \mu_{1}=4.73004074
\end{aligned}
$$

We note here that the solution (34) are approximate ones. These are in fact Galerkin trial functions. Following a proce- dure similar to that in case (i), we obtain the Eqs. 27)-(31) with i taking the value 2 . The various quantities concerning case (ii) are:

$U_{2}=\frac{M P_{1} \delta^{6} \Lambda}{4 A^{5} \pi^{4} P_{4}^{2}\left(R_{1_{c}}\right)_{2}}, \quad r_{\Phi_{2}}=\frac{R_{2}}{\left(R_{1_{c}}\right)_{2}} \frac{M}{\phi}, \quad r_{2}=\frac{R_{1}}{\left(R_{1_{c}}\right)_{2}}$

$\left(R_{1_{c}}\right)_{2}=\frac{\Lambda \mu_{1}^{4}\left[\left(A^{4}+1\right) P_{2}+2 A^{2} P_{3}\right]+A^{2} \sigma^{2} \mu_{1}^{2} P_{1}\left(A^{2}+1\right)}{4 A^{5} \pi^{2} \mu_{1}^{2} P_{4}^{2} / M \delta^{2}}$

$P_{1}=\frac{g_{1}\left(\mu_{1}\right)}{2 \mu_{1}^{2}}\left[\frac{\left(\mu_{1}-\sin \mu_{1}\right)}{\cos ^{2}\left(\mu_{1} / 2\right)}+\frac{\left(\mu_{1}-\sinh \mu_{1}\right)}{\cosh ^{2}\left(\mu_{1} / 2\right)}\right]>0$,

$P_{2}=\left[\frac{g_{3}\left(\mu_{1}\right)\left(\sin \mu_{1}-\mu_{1}\right)+g_{2}\left(\mu_{1}\right)\left(\sinh \mu_{1}-\mu_{1}\right)}{2 \mu_{1} \sqrt{g_{2}\left(\mu_{1}\right) g_{3}\left(\mu_{1}\right)} \cos \left(\mu_{1} / 2\right) \cosh (\mu / 2)}\right]^{2}$,

$P_{3}=\left[\frac{g_{3}\left(\mu_{1}\right)\left(\sin \mu_{1}+\mu_{1}\right)-g_{2}\left(\mu_{1}\right)\left(\sinh \mu_{1}+\mu_{1}\right)}{2 \mu_{1} \sqrt{g_{2}\left(\mu_{1}\right) g_{3}\left(\mu_{1}\right)} \cos \left(\mu_{1} / 2\right) \cosh \left(\mu_{1} / 2\right)}\right]^{2}$,

$g_{1}\left(\mu_{1}\right)=\frac{\mu_{1}+\sin \mu_{1}}{1+\cos \mu_{1}}-\frac{\mu_{1}+\sinh \mu_{1}}{1+\cosh \mu_{1}}$,

$g_{2}\left(\mu_{1}\right)=1+\cos \mu_{1}, g_{3}\left(\mu_{1}\right)=1+\cosh \mu_{1}$,

$P_{4}=\frac{16 \pi^{2} \mu_{1}^{3}}{\left(\pi^{4}-\mu_{1}^{4}\right)^{2}}, P_{5}=\frac{32 \pi^{3} \mu_{1}^{3}\left(39 \pi^{4}+\mu_{1}^{4}\right)}{\left(\pi^{4}-\mu_{1}^{4}\right)^{2}\left(81 \pi^{4}-\mu_{1}^{4}\right)}$.

On obtaining the Lorenz model, we now move on to get the Ginzburg-Landau model using a regular perturbation method.

\subsection{Derivation of Ginzburg-Landau Equation}

Let

$$
L=\left[\begin{array}{ccccc}
-a P r & a P r & 0 & -a \operatorname{Pr} r_{\Phi_{i}} & 0 \\
a r_{i_{0}} & -a & 0 & 0 & 0 \\
0 & 0 & -a b^{*} & 0 & 0 \\
a r_{i_{0}} & 0 & 0 & \frac{-a r_{i_{0}}}{\phi L e} & 0 \\
0 & 0 & 0 & 0 & \frac{-a b^{*} r_{i_{0}}}{\phi L e}
\end{array}\right], I_{k}=\left[\begin{array}{c}
X_{i_{k}} \\
Y_{i_{k}} \\
Z_{i_{k}} \\
E_{i_{k}} \\
F_{i_{k}}
\end{array}\right]
$$

where $\mathrm{k}=1,2,3$.

The following perturbation expansions are used in the system 27)-31 along with a small time scale, $\tau^{*}=\varepsilon^{2} \tau_{1}$ :

$$
\left.\begin{array}{rl}
X_{i} & =\varepsilon X_{i_{1}}+\varepsilon^{2} X_{i_{2}}+\varepsilon^{3} X_{i_{3}}+\ldots \\
Y_{i} & =\varepsilon Y_{i_{1}}+\varepsilon^{2} Y_{i_{2}}+\varepsilon^{3} Y_{i_{3}}+\ldots \\
Z_{i} & =\varepsilon Z_{i_{1}}+\varepsilon^{2} Z_{i_{2}}+\varepsilon^{3} Z_{i_{3}}+\ldots \\
E_{i} & =\varepsilon E_{i_{1}}+\varepsilon^{2} E_{i_{2}}+\varepsilon^{3} E_{i_{3}}+\ldots \\
F_{i} & =\varepsilon F_{i_{1}}+\varepsilon^{2} F_{i_{2}}+\varepsilon^{3} F_{i_{3}}+\ldots \\
r_{i} & =r_{i_{0}}+\varepsilon r_{i_{1}}+\varepsilon^{2} r_{i_{2}}+\varepsilon^{3} r_{i_{3}}+\ldots
\end{array}\right\} .
$$

On substituting Eq. 37 in Eqs. 27)-(31) and equating the coefficients of like powers of $\varepsilon$ on both sides, we obtain 
First-order system: $\quad L I_{1}=0$,

Second-order system: $L I_{2}=\left[\begin{array}{lllll}R_{21} & R_{22} & R_{23} & R_{24} & R_{25}\end{array}\right]^{T}(39)$

Third-order system: $L I_{3}=\left[\begin{array}{lllll}R_{31} & R_{32} & R_{33} & R_{34} & R_{35}\end{array}\right]^{T}(40)$

where

$$
\begin{aligned}
& R_{21}=0, R_{22}=a X_{i_{1}} Z_{i_{1}}, R_{23}=-a X_{i_{1}} Y_{i_{1}}, \\
& \left.R_{24}=a r_{i_{0}} X_{i_{1}} F_{i_{1}}, R_{25}=-a r_{i_{0}} \frac{M^{2}}{\phi^{2}} X_{i_{1}} E_{i_{1}},\right\} \\
& R_{31}=U_{i} \frac{d X_{i_{1}}}{d \tau^{*}}, R_{33}=\frac{d Z_{i_{1}}}{d \tau^{*}}-a X_{i_{1}} Y_{i_{2}}-a X_{i_{2}} Y_{i_{1}}, \\
& R_{32}=\frac{d Y_{i_{1}}}{d \tau^{*}}-a r_{i_{2}} X_{i_{1}}+a X_{i_{1}} Z_{i_{2}}+a X_{i_{2}} Z_{i_{1}}, \\
& R_{34}=a\left[\frac{r_{i_{0}}}{a} \frac{d E_{i_{1}}}{d \tau^{*}}-r_{i_{2}} X_{i_{1}}+\frac{N_{A}}{L e M} Y_{i_{1}}+\frac{r_{i_{2}}}{L e \phi} E_{i_{1}}\right. \\
& \left.+r_{i_{0}}\left(X_{i_{1}} F_{i_{2}}+X_{i_{2}} F_{i_{1}}\right)\right], R_{35}=\frac{a b^{*}}{L e \phi}\left[N_{A} Z_{i_{1}}+r_{i_{2}} F_{i_{1}}\right] \\
& +r_{i_{0}}\left[\frac{d F_{i_{1}}}{d \tau^{*}}-\frac{a M^{2}}{\phi^{2}}\left(X_{i_{1}} E_{i_{2}}+X_{i_{2}} E_{i_{1}}\right)\right] .
\end{aligned}
$$

On solving Eqs. [38- 40], we obtain the following solutions:

First-order solution:

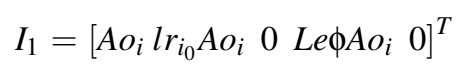

Second-order solution:

$$
I_{2}=\left[\begin{array}{lll}
0 & 0 & \frac{r_{i_{0}}}{b} A o_{i}^{2} 0 \frac{-L e^{2} M^{2}}{b} A o_{i}^{2}
\end{array}\right]^{T}(44)
$$

where $A o_{i}$ is an arbitrary function of $\tau^{*}$. Now to determine the thermal scaled Rayleigh number and the amplitude of convection $A o_{i}$ we make use of the Fredholm solvability condition as:

$$
\sum_{j=1}^{5} \hat{I}_{1}^{T} R_{k j}=0, \quad(k=2,3)
$$

where $\hat{I}_{1}^{T}$ is the solution of adjoint of the first-order system . For $\mathrm{k}=2$, we get $r_{i_{1}}=0$ and $\mathrm{k}=3$ gives the following Ginzburg-Landau equation.

$$
\frac{d A o_{i}}{d \tau^{*}}-F_{1_{i}} A o_{i}+F_{2_{i}}\left(A o_{i}\right)^{3}=0,
$$

where

$F_{1_{i}}=\frac{a \operatorname{Pr}\left[1+\phi r_{\Phi_{i}} N_{A} / M\right]}{U_{i}+\operatorname{Pr}-L e^{2} \phi^{2} r_{\Phi_{i}} P r}, F_{2_{i}}=\frac{a \operatorname{Pr} / b\left[1-L e^{3} M^{2} \phi r_{\Phi_{i}}\right]}{U_{i}+\operatorname{Pr}-L e^{2} \phi^{2} r_{\Phi_{i}} \operatorname{Pr}}$.
Since $r_{i_{2}}$ is a correction to the scaled critical thermal Rayleigh number, we choose $r_{i_{2}}=1$ for simplicity. Now on solving Eq. (46) with initial condition $A o_{i}(0)=1$, we get an expression for amplitude of convection as:

$$
A o_{i}=\frac{1}{\sqrt{F_{3_{i}}+\left(1-F_{3_{i}}\right) e^{-2 F_{1_{i}} \tau^{*}}}} \quad \text { and } \quad F_{3_{i}}=\frac{F_{2_{i}}}{F_{1_{i}}}
$$

Having obtained the expression for the amplitude of convection in the unsteady case, we now move on to discuss about the stability boundaries of the system.

\subsection{Stability Boundaries}

To draw the stability boundaries, we consider the linearised form of the Lorenz model in Eqs. 277- 31):

$$
\left.\begin{array}{rl}
U_{i} \frac{d X_{i}}{d \tau_{1}} & =a \operatorname{Pr}\left[Y_{i}-X_{i}-r_{\Phi_{i}} E_{i}\right], \\
\frac{d Y_{i}}{d \tau_{1}} & =a\left[r_{i} X_{i}-Y_{i}\right], \\
\frac{d E_{i}}{d \tau_{1}} & =a\left[X_{i}-\frac{\varepsilon^{2} N_{A}}{L e} \frac{1}{r_{i} M} Y_{i}-\frac{1}{L e} \frac{1}{\phi} E_{i}\right] \cdot
\end{array}\right\}
$$

Assume the following solution for $X_{i}, Y_{i}, E_{i}$ :

$$
X_{i}=X_{i_{0}} e^{\Omega \tau_{1}} Y_{i}=Y_{i_{0}} e^{\Omega \tau_{1}}, E_{i}=E_{i_{0}} e^{\Omega \tau_{1}}, \Omega=\Omega_{r}+j \Omega_{j}
$$

where $j=\sqrt{-1}$. Substitution of Eq. (49) in Eq. (48) gives the following characteristic equation which is cubic in $\Omega$, whose real part represents the growth rate and imaginary part represents the frequency of oscillation:

$$
\left.\begin{array}{c}
\Omega^{3} U_{i}+\Omega^{2} a\left[\operatorname{Pr}+U_{i}+\frac{U_{i}}{L e \phi}\right]+\Omega a^{2} \operatorname{Pr}\left[1-\frac{8 \pi^{4} R_{1}^{*}}{\left(R_{1_{c}}\right)_{i}}\right. \\
\left.+\frac{R_{2}}{\left(R_{1_{c}}\right)_{i}} \frac{M}{\phi}+\frac{1}{L e \phi}\left(1+\frac{U_{i}}{P r}\right)\right]+\frac{P r}{L e \phi} a^{3} \\
-\frac{P r}{L e \phi} a^{3}\left[\frac{R_{2}}{\left(R_{\left.1_{c}\right)_{i}}\right.}\left(\varepsilon^{2} N_{A}-M L e\right)+8 \pi^{4} \frac{R_{1}^{*}}{\left(R_{1_{c}}\right)_{i}}\right]=0, \\
R_{1}^{*}=R_{1} /\left(8 \pi^{4}\right) .
\end{array}\right\}
$$

The lines

Stationary

$$
X_{3} U:\left(R_{1}^{*}\right)_{i}^{S}=\frac{\left(R_{1_{c}}\right)_{i}}{8 \pi^{4}}+\frac{R_{2}}{8 \pi^{4}}\left(M L e-\varepsilon^{2} N_{A}\right),
$$

Oscillatory

$$
\left.\begin{array}{c}
X_{4} W:\left(R_{1}^{*}\right)_{i}^{O}=\frac{\left(R_{1_{c}}\right)_{i}}{8 \pi^{4}}\left\{\left(1+\frac{U_{i}}{L e \phi P r}\right)\right. \\
\left.\left[1+\frac{1}{L e \phi}+\frac{P r}{P r+U_{i}} \frac{R_{2}}{\left(R_{1_{c}}\right)_{i}} \frac{M}{\phi}\right]\right\},
\end{array}\right\}
$$


Copyright (cre the stability boundaries between unstable(fully developed) region and stable region of the system. Along the line $X_{3} U$ one of the roots of the Eq. A1] is zero which gives the value of small amplitude Rayleigh number. On the line $X_{4} W$, the complex root has zero real part which allows the system for oscillatory convection. The line $R_{1}^{*}=R_{2}$ gives the static condition in the system, i.e., the destabilizing temperature variation will be compensated by the stabilizing concentration variation. Eliminating the real part, $\Omega_{r}$, in Eq. (A1) we get a cubic equation in $\Omega_{j}$. Limitation of $\Omega_{j}$ to zero leads to the line $X_{1} V$. The critical Rayleigh number of a nonlinear stability analysis required for plotting the stability boundaries is discussed in section 6.

\subsection{Subcritical Instabilities}

On solving the Eqs. 27)-(31) at steady state, we get the expression for the amplitudes in the form:

$$
\left.\begin{array}{l}
X_{i}^{2}=\frac{-Q_{2_{i}}+\sqrt{Q_{2_{i}}^{2}-4 Q_{1_{i}} Q_{3_{i}}}}{2 Q_{1_{i}}}, Y_{i}=\frac{r_{i} b^{*} X_{i}}{b^{*}+X_{i}^{2}}, \\
Z_{i}=\frac{r_{i} X_{i}^{2}}{b^{*}+X_{i}^{2}}, \quad E_{i}=\frac{X_{i}}{r_{\Phi_{i}}}\left(\frac{r_{i} b^{*}}{b^{*}+X_{i}^{2}}-1\right), \\
F_{i}=-X_{i}^{2}\left[\frac{\varepsilon^{2} N_{A}}{b^{*}+X_{i}^{2}}-\frac{L e M^{2}}{r_{\Phi_{i}} \phi}\left(\frac{r_{i}}{b^{*}+X_{i}^{2}}-\frac{1}{b^{*}}\right)\right],
\end{array}\right\}
$$

where $Q_{1_{i}}=\frac{L e M^{2}}{\phi r_{\Phi_{i}} b^{*}}, Q_{2_{i}}=\frac{L e M^{2}}{\phi r_{\Phi_{i}}}\left(1-r_{i}\right)+\varepsilon^{2} N_{A}+1+$ $\frac{1}{\phi r_{\Phi_{i}} L e}, Q_{3_{i}}=b^{*}\left(1-\frac{\varepsilon^{2} N_{A}}{L e} \frac{1}{M}\right)+\frac{b^{*}}{\phi L e r_{\Phi_{i}}}\left(1-r_{i}\right)$.

On equating the discriminant of $X_{i}^{2}$ in Eq. (54) to zero, we obtain the line of stability for finite amplitude thermal Rayleigh number as:

$$
X_{2} V:\left(R_{1}\right)_{i}^{F}=\left(R_{1}\right)_{i}^{S}-\left(s_{i}+t\right)^{2}
$$

where $t=\sqrt{\frac{R_{2}(1+\text { Le } M)\left(\text { Le } M-1-\varepsilon^{2} N_{A}\right)}{\text { Le } M}}$ and $s_{i}=$ $\frac{\sqrt{\left(R_{1_{c}}\right)_{i}}}{\text { Le } M}$.

Having found the expressions for amplitudes of convection and thermal Rayleigh number we now move on to find the heat transport in terms of Nusselt number.

\section{Heat Transport}

The thermal Nusselt number is defined as:

$$
\mathrm{Nu}=1+\left[-k \int_{\frac{-1}{2}}^{\frac{1}{2}}\left(\frac{\partial \Theta}{\partial Z}\right) d X /-k_{\mathrm{bl}_{\mathrm{eff}}} \int_{\frac{-1}{2}}^{\frac{1}{2}}\left(\frac{d \Theta_{b}}{d Z}\right) d X\right]_{Z=-\frac{1}{2}}
$$

On substituting Eqs. (13), 26) and (33) in Eq. (56), using the scaling (18) and Eqs. 32, (35), we get an expression for Nusselt number for different boundaries as:

Case (i): Free-Free

$$
\mathrm{Nu}\left(\tau^{*}\right)=1+\frac{k}{k_{\mathrm{bl}_{\mathrm{eff}}}} \frac{2}{b^{*}} r_{1_{0}}\left[1-\frac{r_{1_{0}}}{r_{1}}\right] A o_{1}^{2}\left(\tau^{*}\right)
$$

Case (ii): Rigid-Rigid

$$
\mathrm{Nu}\left(\tau^{*}\right)=1+\frac{k}{k_{\mathrm{bl}_{\mathrm{eff}}}} \frac{2}{b^{*}} \frac{\pi P_{4}}{P_{5}} r_{2_{0}}\left[1-\frac{r_{2_{0}}}{r_{2}}\right] A o_{2}^{2}\left(\tau^{*}\right)
$$

where $A o_{i}^{2}$ and $X_{i}^{2}(i=1,2)$ are given in (47) and one of the expressions in Eq. (54) respectively.

After having obtained the expressions for small and finite amplitude Rayleigh numbers and the Nusselt number in the case of both stress-free and rigid boundaries, we now move on to the discussion of results.

\section{Results and Discussion}

Rayleigh-Bénard convection in Newtonian nanoliquids confined in a porous enclosure is studied using modified Buongiorno - Brinkman model. The values of thermophysical properties of water (base liquid) and copper nanoparticles are taken from ( [3], [22], [48]) and those of 30\% reinforced polycarbonate glass fiber porous material [49] is documented in Tab. 3. The values of thermophysical properties of nanoliquids are taken from Siddheshwar et al. [22] and those of nanoliquid-saturated porous medium is tabulated in Tab. 2 These are calculated using the phenomenological laws or traditional mixture theory reported in Eqs. (6)-(8). The tab-

Table 1: Thermophysical properties of $30 \%$ reinforced polycarbonate glass fiber porous material at $300 \mathrm{~K}$.

\begin{tabular}{|c|c|c|c|c|c|}
\hline$\rho_{0_{p m}}$ & $c_{p m}$ & $k_{p m}$ & $\chi_{p m} \times 10^{7}$ & $\beta_{1_{p m}} \times 10^{5}$ & $\phi$ \\
\hline 1430 & 1130 & 0.24 & 1.48524 & 3.5 & 0.88 \\
\hline
\end{tabular}

Table 2: Thermophysical properties of nanoliquid-saturated porous medium with $\alpha=0.06$ and $\phi=0.88$ at $300 \mathrm{~K}$.

\begin{tabular}{|c|c|c|c|c|c|}
\hline$\rho_{0}$ & $\mu \times 10^{3}$ & $c$ & $k$ & $\chi \times 10^{7}$ & $\beta_{1} \times 10^{5}$ \\
\hline 1468.06 & 1.4288 & 2603.66 & 0.6710 & 1.7555 & 12.7441 \\
\hline
\end{tabular}

ulated values of the thermophysical quantities reported by Siddheshwar et al. [22] and those tabulated in Tabs. 3 and 2 reveal the following results:

$\rho_{0_{b l}}<\rho_{0_{p m}}<\rho_{0}<\rho_{0_{n l}}<<\rho_{0_{n p}}$,

$k_{p m}<k_{b l}<k<k_{n l}<<k_{n p}, \mu_{b l}<\mu_{n l}<\mu$,

$\beta_{1_{b l}}>\beta_{1_{n l}}>\beta_{1}>\beta_{1_{p m}}>\beta_{1_{n p}}, c_{b l}>c_{n l}>c>c_{p m}>c_{n p}$. 
in order to understand the advanced onset situation in nanoliquid due to the presence of nanoparticles, we rewrite $R_{1}$ given in Eq. 22, as $R_{1}=F \cdot R_{1_{b l}}$,

where $F=\frac{\rho_{0} \beta_{1}}{\left(\rho_{0} \beta_{1}\right)_{b l}} \frac{\mu_{b l}}{\mu_{n l}} \frac{\chi_{b l}}{\chi}, \quad R_{1_{b l}}=\frac{\left(\rho_{0} \beta_{1}\right)_{b l} \Delta T g b^{3}}{\mu_{b l} \chi_{b l}}$.

The documented values of thermophysical properties reveal that $\left(\rho_{0} \beta_{1}\right)_{b l}>\rho_{0} \beta_{1}, \mu_{n l}>\mu_{b l}, \chi>\chi_{b l}$ and hence the factor, $\mathrm{F}$, is less than unity for water-copper nanoliquid. Since $F$ decreases with increase in volume fraction we may infer that $R_{1}<R_{1_{b l}}$.

Before we move onto discuss the results of our study we note that the parameters concerning a porous medium were decided upon based on the fact that the medium is sparsely packed and hence the range of permissible value of Darcy number is $0<\sigma^{2}<10^{2}$. This is typically the value chosen in Rayleigh-Bénard convection of Newtonian liquids in porous media. In our study thermal equilibrium is assumed between the porous medium made of $30 \%$ reinforced polycarbonate glass fiber spheres and the nanoliquid and this is valid when the thermal conductivity of the two differs considerably. In this problem we do not use aluminium foam as porous material which has very high thermal conductivity compared to the nanoliquid. Further thermal equilibrium is justified when the range of temperatures considered are in the neighbourhood of the ambient temperature.

Keeping in mind that uni-cellular convection in enclosures is being studied, aspect ratios in the maximum range $[0.8,2.05]$ are considered to cover the results of square, shallow and tall porous enclosures with free-free and rigid-rigid boundaries.

Following Baines and Gill [50], we have plotted the stability diagram for nanoliquid-saturated porous medium confined in an enclosure for both free-free and rigid-rigid boundaries.

In Figs. 2 and 3 we observe that the oscillatory stability curve is in the fully developed flow region. This is practically not possible. Thus we conclude that oscillatory convection is not possible in case of dilute concentration of nanoparticles thereby proving the validity of the principle of exchange of stability(PES).

In region I, the solution of Eq. A1 has a negative real part. Thus the points in region I are stable. In region II, the root has a positive real part, thus the system has unstable direct modes. Region III is bounded by the lines $X_{3} U$ and $X_{2} Y$ which represents small and finite amplitude Rayleigh number curves respectively. From the graph it is clear that the finite amplitude Rayleigh number curve lies below the small amplitude Rayleigh number curve forming a subcritical region, which is shaded in the graph. This confirms the existence of subcritical motions in the system. Region IV is the fully developed flow region where the system is unstable.

In Fig. 2 we have plotted stability diagram for different types of enclosures for both free-free and rigid-rigid boundary conditions. We observe that the stability region is reduced i.e., the onset of convection is advanced in the case of tall enclosure compared to that in the case of a shallow enclosure. The result is true irrespective of the boundary combination considered.
Figure 3 is the stability diagram for nanoliquid and nanoliquid-saturated porous medium for both free-free and rigid-rigid boundary conditions. Here we notice that stability region is enlarged in case of nanoliquid-saturated porous medium compared to that in the case of a nanoliquid(without porous matrix). Thus we conclude that presence of porous medium has a stabilizing effect on the onset of convection.

The main emphasis of the present paper is on the heat transport in nanoliquid-saturated porous medium and the same is quantified in terms of Nusselt number. For different values of aspect ratio, the Nusselt number is plotted in Figs. $4 \mathrm{a}$ and $4 \mathrm{~b}$ for the two boundary conditions and it can be seen that the heat transport increases with increase in aspect ratio. From the plot we may infer that enhanced heat transport in tall and square porous enclosures is more pronounced compared to that in shallow enclosures.

Figures 4c and 4d are plots of Nusselt number for different values of volume fraction, $\alpha$. The plots show an increase in the heat transport with increase in $\alpha$. Figures $4 \mathrm{e}, 4 \mathrm{~h}$ show that the heat transport decreases with individual and collective increases in $\Lambda$ and $\sigma^{2}$. This shows the reduced heat transport in the presence of porous medium due to the low thermal conductivity of the medium. Computations reveal the reduced heat transport situation in nanoliquid-saturated porous enclosure when compared with nanoliquid confined in an enclosure without porous matrix.

Figure 5 is the plot of amplitudes in both linear and nonlinear cases for different values of $\sigma^{2}$ with $A o_{i}(0)=0.001$. We observe here that the amplitude curves in linear case is unbounded and the cubic non-linear term in the GinzburgLandau equation helps in bounding the solution. This nature of the amplitudes in the Ginzburg-Landau model is inherited from the Lorenz model.

We know that the difference between KVLM and MBBM is that the two-phase model incorporates the effects of thermophoresis and Brownian diffusion. So if we neglect these effects in the MBBM then we arrive at the KVLM as a limiting case.

It is pretty clear from Eq. (52) that $\left(R_{1}\right)_{i}^{M B B M}=\left(R_{1}\right)_{i}^{K V L M}+R_{2}\left[M L e-\varepsilon^{2} N_{A}\right]$ for the two boundary combinations. This means that the KVL model underpredicts the eigenvalue when compared with that predicted by the MBBM and also that the correction to the KVL model results is entirely due to the incorporation of Brownian and thermophoresis effects and is independent of the boundary combination we use.

The results of the problem have possible implication in heat storage and heat removal systems. Nanoparticles provide the heat removal mechanism due to their high thermal conductivity and porous medium helps in the retainment of energy due to its low thermal conductivity.

\section{Validation of Results}

Validation of results of the present model is done by comparing the results of the present model with those of Elhajjar et al. [36] for rigid-rigid boundaries. Good agreement of the present result with those of Elhajjar et al. [36] is 

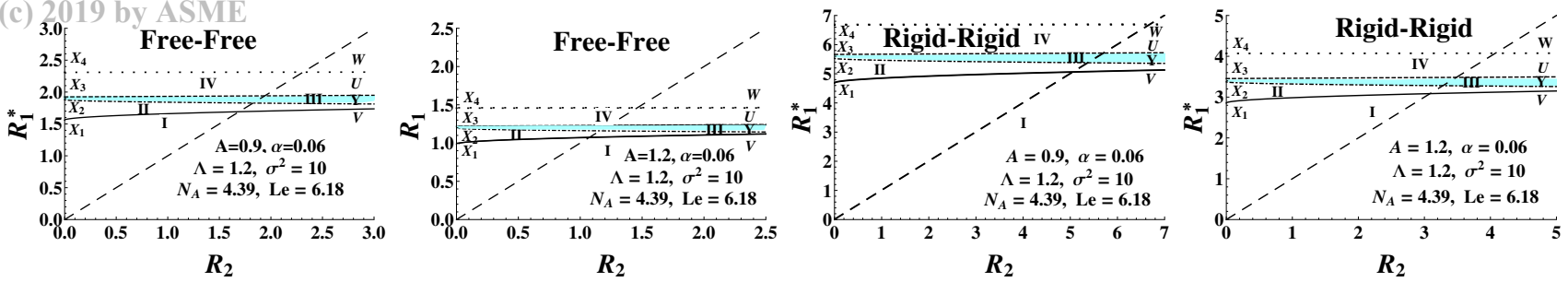

Fig. 2: Stability diagrams for water-copper saturated porous medium in shallow and tall enclosures.
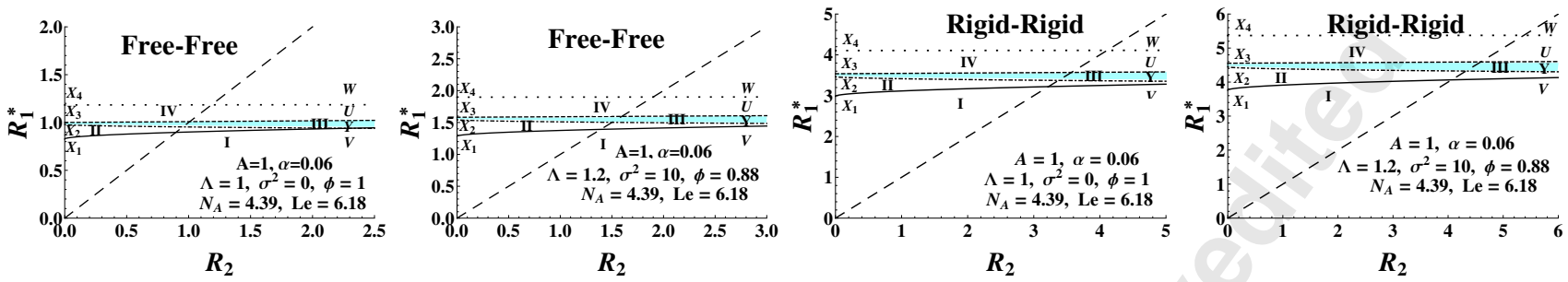

Fig. 3: Stability diagrams for water-copper nanoliquid and water-copper nanoliquid-saturated porous medium.

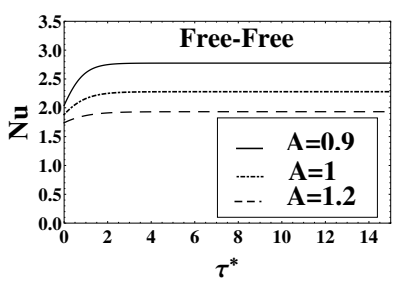

(a) $\alpha=0.06, \Lambda=1.2, \sigma^{2}=10$.

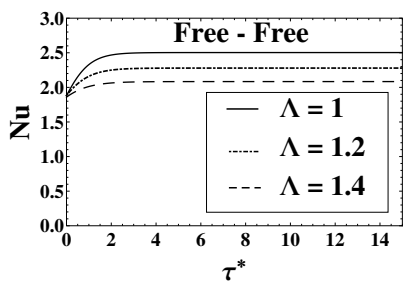

(e) $\mathrm{A}=1, \alpha=0.06, \sigma^{2}=10$.

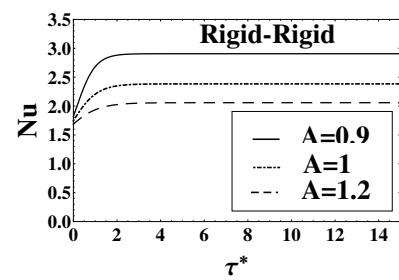

(b) $\alpha=0.06, \Lambda=1.2, \sigma^{2}=10$.

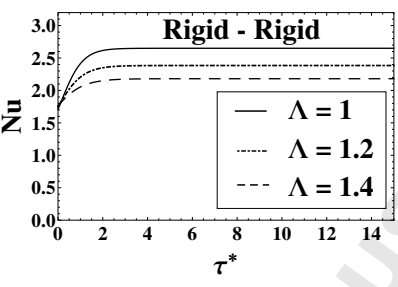

(f) $\mathrm{A}=1, \alpha=0.06, \sigma^{2}=10$.

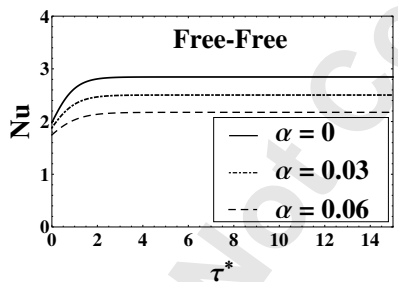

(c) $\mathrm{A}=1, \Lambda=1.2, \sigma^{2}=10$.

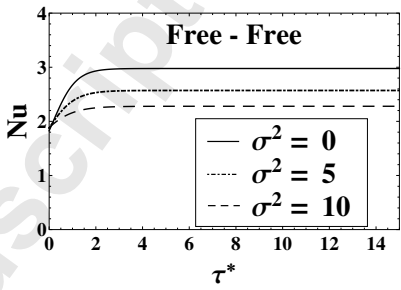

(g) $\mathrm{A}=1, \alpha=0.06, \Lambda=1.2$.

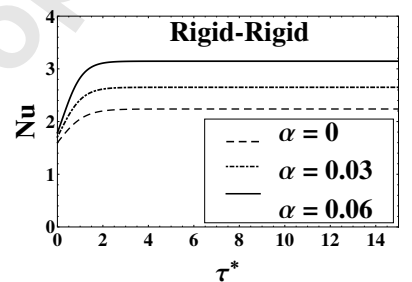

(d) $\mathrm{A}=1, \Lambda=1.2, \sigma^{2}=10$.

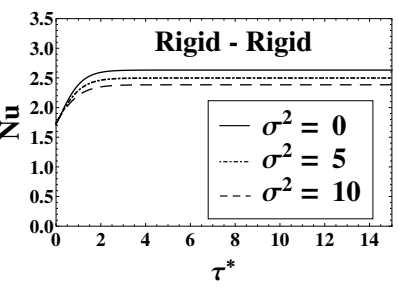

(h) $\mathrm{A}=1, \alpha=0.06, \Lambda=1.2$.

Fig. 4: Plot of Nusselt number, Nu, versus $\tau^{*}$ for different values of $\mathrm{A}, \alpha, \Lambda$ and $\sigma^{2}$.
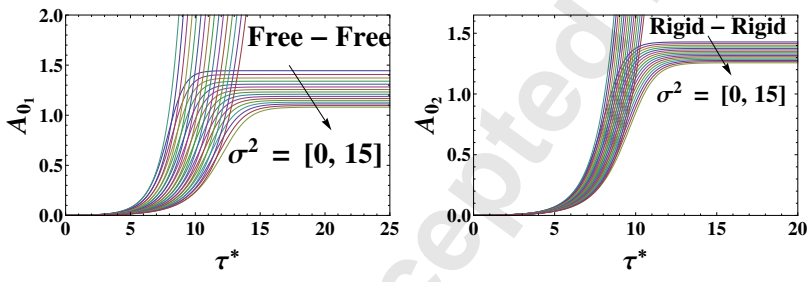

Fig. 5: Plot of linear and non-linear amplitudes, $A_{0}$ versus $\tau^{*}$ for different values of $\sigma^{2}$ and $A=1, \alpha=0.06, \Lambda=1.2$.

observed for the rectangular enclosure $(\mathrm{A}=0.8688$ for watercopper) with an average \% error of 0.0032 for $R_{1}=5000$. Disagreement of the result of Elhajjar et al. [36] for $R_{1}<$ 5000 on Nusselt number with volume fraction is because of their incorrect modelling of specific heat and thermal expansion coefficient. Thermodynamically correct results are ob-
Table 3: Comparison of the results of the paper with those of Elhajjar et al. [36], for $R_{1}=5000$ in the case of a rectangular enclosure filled with water-copper nanoliquid with $\alpha=0.08$.

\begin{tabular}{|c|c|c|c|}
\hline Quantity & Elhajjar et al. [36] & Present paper & \% error \\
\hline $\mathrm{Nu}$ & 1.5186 & 1.51865 & 0.0032 \\
\hline
\end{tabular}

tained in the present study with the redefined definition of specific heat and thermal expansion coefficient as in Eq. (7).

Since the solution (34) is an approximate one, the Rayleigh number in Eq. (52) with $\mathrm{i}=2$ is approximate too. To gain confidence in the approximate values of critical Rayleigh number in case (ii), we obtained the same by the shooting method. The maximum percentage error for all parameters' combination was found to be $2.18 \%$. The results of linear stability are thus quite accurate in this case also. 


\section{Copyright (g) 2019 housion}

1. Addition of nanoparticles into the system effectively increases the thermal conductivity. Thus, a base liquid with nanoparticles can serve as a coolant.

2. Presence of porous medium reduces the thermal conductivity of the system. Thus, porous medium can act as a heat storage system.

3. The following conclusions on the parameters' influence on onset of convection, $\left(R_{1}\right)_{i}^{S}$, have been arrived at using Eq. 52 and stability diagrams given in Figs. 2 and 3 . The corresponding ones on heat transport, $\mathrm{Nu}$, can be seen in the Nusselt number plots of Figs. 4 .

(a) $\frac{d}{d \alpha}\left(R_{1}\right)_{i}^{S}<0 \Rightarrow \frac{d}{d \alpha}(N u)>0 ; \frac{d}{d A}\left(R_{1}\right)_{i}^{S}<0 \Rightarrow$ $\frac{d}{d A}(N u)>0$

(b) $\frac{d}{d \Lambda}\left(R_{1}\right)_{i}^{S}>0 \Rightarrow \frac{d}{d \Lambda}(N u)<0 ; \frac{d}{d \sigma^{2}}\left(R_{1}\right)_{i}^{S}>0 \Rightarrow$ $\frac{d}{d \sigma^{2}}(N u)<0$

(c) $\frac{d}{d R_{2}}\left(R_{1}\right)_{i}^{S}<0 \Rightarrow \frac{d}{d R_{2}}(N u)>0 ; \frac{d}{d L e}\left(R_{1}\right)_{i}^{S}>0 \Rightarrow$ $\frac{d}{d L e}(N u)<0 ; \frac{d}{d N_{A}}\left(R_{1}\right)_{i}^{S}>0 \Rightarrow \frac{d}{d N_{A}}(N u)<0$.

4. Oscillatory convection is not possible.

5. $\left(R_{1}\right)_{i}^{F}<\left(R_{1}\right)_{i}^{S}$. This confirms the existence of subcritical motions.

6. KVL single-phase model is a limiting case of MBB twophase model.

7. The assumption of unicellular convection is valid only for the aspect ratios ranging between $0.85-2.05$ in the case of stress-free boundaries and 0.85-1.84 in the case of rigid boundaries. In the case of aspect ratio taking values less than 0.85 , multicellular convection manifests and not unicellular as assumed in the paper. The study for very small A can be made by following the classical Rayleigh-Bénard analysis (with lateral boundary effects taken off). Large values of A concern physically unrealistic situations.

8. Convective activity is more pronounced in tall enclosures compared to the other two types of enclosures.

9. Computations reveal the following general results:

(i) $N u_{n l}>N u$ and (ii) $N u>N u_{b l_{\text {eff }} \text {. }}$.

10. Non-linear term in the Ginzburg-Landau equation ensures a bounded solution.

\section{Acknowledgment}

Lakshmi is thankful to the Department of Backward Classes Welfare, Government of Karnataka and to the Bangalore University for supporting her research. The authors are grateful to the reviewers for helpful comments.

\section{Nomenclature \\ Latin Symbols \\ A aspect ratio \\ c effective specific heat capacity $(\mathrm{J} /[\mathrm{Kg}-\mathrm{K}])$}

C concentration of nanoparticles $(\mathrm{Kg})$

$D_{B} \quad$ modified Brownian diffusion coefficient $\left(\mathrm{m}^{2} / \mathrm{s}\right)$

$D_{T}$ modified thermophoresis diffusion coefficient $\left(\mathrm{m}^{2} / \mathrm{s}\right)$

$d_{n p} \quad$ diameter of nanoparticles $(\mathrm{m})$

$E_{i}, F_{i}$ amplitudes of non-linear convection(m)

$\mathrm{g}=(0,0,-\mathrm{g}) \quad$ acceleration due to $\operatorname{gravity}\left(\mathrm{m} / \mathrm{s}^{2}\right)$

$\mathrm{k}$ effective thermal conductivity $(\mathrm{W} /[\mathrm{m}-\mathrm{K}])$

$\mathrm{K}$ permeability $\left(\mathrm{m}^{2}\right)$

$K_{B} \quad$ Boltzmann constant $(\mathrm{J} / \mathrm{K})$

Le Lewis number

$\mathrm{M}$ ratio of specific heats

$N_{A}$ modified diffusivity ratio

$\mathrm{Nu}$ Nusselt number

$\mathrm{P}$ dynamic pressure $(\mathrm{Pa})$

Pr modified Prandtl number

q velocity of the nanoliquid( $\mathrm{m} / \mathrm{s})$

$R_{1}, R_{2}$ thermal and concentration Rayleigh number

$\mathrm{t}$ dimensional time(s)

$\mathrm{T}$ dimensional temperature $(\mathrm{K})$

$\mathrm{u}, \mathrm{w}$ horizontal and vertical velocity components(m)

$X_{i}, Y_{i}, Z_{i}$ amplitudes of linear convection(m)

$X_{i_{0}}, Y_{i_{0}}, E_{i_{0}}$ infinitesimal amplitudes of convection(m)

$\mathrm{X}, \mathrm{z}$ dimensional horizontal and vertical coordinates

$\mathrm{X}, \mathrm{Z}$ dimensionless horizontal and vertical coordinates

Greek symbols

$\alpha$ volume fraction $(0 \leq \alpha<1)$

$\beta_{1}$ effective thermal expansion coefficient $(1 / \mathrm{K})$

$\beta_{2}$ effective solutal analog of $\beta_{1}(1 / \mathrm{Kg})$

$\chi$ effective thermal diffusivity $\left(\mathrm{m}^{2} / \mathrm{s}\right)$

$\Delta$ difference between values

$\mu$ effective dynamic coeffecient of viscosity $(\mathrm{Kg} /[\mathrm{m}-\mathrm{s}])$

$\Lambda$ Brinkman number

$\phi \operatorname{porosity}(0<\phi \leq 1)$

$\psi$ dimensional form of stream function $\left(\mathrm{m}^{2} / \mathrm{s}\right)$

$\Psi$ non-dimensional form of stream function

$\rho_{0} \quad$ effective density $\left(\mathrm{Kg} / \mathrm{m}^{3}\right)$

$\sigma^{2}$ porous parameter

$\Phi, \Theta$ dimensionless concentration and temperature

$\tau$ dimensionless time

\section{Subscripts and Superscripts}

b basic state

bl, $b l_{\text {eff }}$ base liquid and effective base liquid

c critical

$\mathrm{i}=1,2$ free-free and rigid-rigid boundaries

$\mathrm{nl}, \mathrm{np}, \mathrm{pm}$ nanoliquid, nanoparticle, porous material

0 at reference value

F, S finite and small amplitudes

\section{Appendix}

Justification for neglect of viscous dissipation term in the energy equation

Now consider the energy equation including the viscous dissipation term

$$
M \frac{\partial T}{\partial t}+(\vec{q} \cdot \nabla) T=M \chi \nabla^{2} T+\frac{\mu_{n l}}{\phi\left(\rho_{0} c\right)_{n l}}(\nabla \vec{q})^{2} .
$$


Copyright fo nind the erfect of viscous dissipation term, we make use of order of magnitude analysis:

$$
\frac{\mu_{n l}}{\phi\left(\rho_{0} c\right)_{n l}}(\nabla \vec{q})^{2} \sim \frac{\mu_{n l}}{\phi\left(\rho_{0} c\right)_{n l}} \frac{U^{2}}{h^{2}},
$$

where $\mathrm{U}$ is the non-dimensional form of velocity. In natural convection the viscous force will be balanced by the buoyancy force(terms of $\mu \nabla^{2} \vec{q}$ and $\rho \vec{g}$ in the linear momentum equation). Thus,

$$
\mu \frac{U}{h^{2}} \sim \rho_{0} g \beta_{1} \Theta
$$

Making use of Eq.A3 in Eq. A2, we obtain

$$
\frac{\mu_{n l}}{\left(\rho_{0} c\right)_{n l}} \frac{1}{\phi}(\nabla \vec{q})^{2} \sim \rho_{0} g \beta_{1} \Theta U
$$

Hence the ratio takes the form

$$
\frac{\mu_{n l}(\nabla \vec{q})^{2}}{\phi\left(\rho_{0} c\right)_{n l}(\vec{q} \cdot \nabla) T} \sim \frac{\rho_{0} g h \beta_{1} \phi^{1.5}}{\left(\rho_{0} c\right)_{n l}}
$$

For water, water-copper nanoliquid and water-copper saturated $30 \%$ reinforced polycarbonate glass fiber, the above ratio is of the order $10^{-10}$. Thus the viscous dissipation term has been neglected in the analysis.

\section{References}

[1] Khanafer, K., Vafai, K., and Lightstone, M., 2003. "Buoyancy-driven heat transfer enhancement in a twodimensional enclosure utilizing nanofluids". Int. J. Heat Mass Transfer, 46, pp. 3639-3653.

[2] Buongiorno, J., 2006. "Convective transport in nanofluids". ASME J. Heat Transfer, 128, pp. 240-250.

[3] Siddheshwar, P. G., and Meenakshi, N., 2015. "Amplitude equation and heat transport for Rayleigh-Bénard convection in Newtonian liquids with nanoparticles". Int. J. Appl. Comp. Math., 2, pp. 1-22.

[4] Abu-Nada, E., 2011. "Rayleigh-Bénard convection in nanofluids: effect of temperature dependent properties". Int. J. Therm. Sci., 50, pp. 1720-1730.

[5] Alloui, Z., Vasseur, P., and Reggio, M., 2011. "Natural convection of nanofluids in a shallow cavity heated from below". Int. J. Therm. Sci., 50, pp. 385-393.

[6] Alsabery, A. I., Saleh, H., Hashim, I., and Siddheshwar, P. G., 2016. "Transient natural convection heat transfer in nanoliquid-saturated porous oblique cavity using thermal non-equilibrium model". Int. J. Mech. Sci., 114, pp. 233-245.

[7] Tzou, D. Y., 2008. "Instability of nanofluids in natural convection”. ASME J. Heat Transfer, 130, pp. 0724011-072401-9.
[8] Tzou, D. Y., 2008. "Thermal instability of nanofluids in natural convection". Int. J. Heat Mass Transfer, 51, pp. 2967-2979.

[9] Yang, C., Li, W., Sano, Y., Mochizuki, M., and Nakayama, A., 2013. "On the anomalous convective heat transfer enhancement in nanofluids: a theoretical answer to the nanofluids controversy". ASME J. Heat Transfer, 135, pp. 054504-1-054504-9.

[10] Sheikholeslami, M., Gorji-Bandpy, M., Ganji, D. D., and Soleimani, S., 2014. "Thermal management for free convection of nanofluid using two phase model". J. Mol. Liq., 194, pp. 179-187.

[11] Bhadauria, B. S., and Agarwal, S., 2011. "Convective transport in a nanofluid saturated porous layer with thermal non equilibrium model". Transp. Porous Media, 88, pp. 107-131.

[12] Agarwal, S., Rana, P., and Bhadauria, B. S., 2014. "Rayleigh-Bénard convection in a nanofluid layer using a thermal nonequilibrium model". ASME J. Heat Transfer, 136, pp. 122501-1-122501-14.

[13] Nield, D. A., and Kuznetsov, A. V., 2009. "Thermal instability in a porous medium layer saturated by a nanofluid". Int. J. Heat Mass Transfer, 52, pp. 57965801.

[14] Kuznetsov, A. V., and Nield, D. A., 2010. "Effect of local thermal non-equilibrium on the onset of convection in a porous medium layer saturated by a nanofluid". Transp. Porous Media, 83, pp. 425-436.

[15] Kuznetsov, A. V., and Nield, D. A., 2011. "The effect of local thermal non-equilibrium on the onset of convection in a porous medium layer saturated by a nanofluid: Brinkman model". J. Porous Media, 14, pp. 285-293.

[16] Khalili, E., Saboonchi, A., and Saghafian, M., 2017. "Experimental study of nanoparticles distribution in natural convection of $\mathrm{Al}_{2} \mathrm{O}_{3}$-water nanofluid in a square cavity". Int. J. Therm. Sci., 112, pp. 82-91.

[17] Yadav, D., Bhargava, R., and Agrawal, G. S., 2012. "Boundary and internal heat source effects on the onset of Darcy-Brinkman convection in a porous layer saturated by nanofluid". Int. J. Therm. Sci., 60, pp. 244254.

[18] Agarwal, S., and Rana, P., 2016. "Convective heat transport by longitudinal rolls in dilute nanoliquid layer of finite depth". Int. J. Therm. Sci., 108, pp. 235-243.

[19] Nield, D. A., and Kuznetsov, A. V., 2011. "The onset of double-diffusive convection in a nanofluid layer". Int. J. Heat Fluid Flow, 32, pp. 771-776.

[20] di Schio, E. R., Celli, M., and Barletta, A., 2014. "Effects of Brownian diffusion and thermophoresis on the laminar forced convection of a nanofluid in a channel". J. Heat Transfer, 136, pp. 022401-1-022401-13.

[21] Barletta, A., Rossi di Schio, E., and Celli, M., 2015. "Convection and instability phenomena in nano-fluidsaturated porous media". Heat Transfer Enhancement with Nanofluids, CRC Press, Boca Raton, FL, pp. 341364.

[22] Siddheshwar, P. G., Kanchana, C., Kakimoto, Y., and Nakayama, A., 2016. "Steady finite-amplitude 
a two-phase model-theoretical answer to the phenomenon of enhanced heat transfer". ASME J. Heat Transfer, 139, pp. 012402-1-012402-8.

[23] Vanaki, S. M., Ganesan, P., and Mohammed, H. A., 2016. "Numerical study of convective heat transfer of nanofluids: A review". Renew. Sustainable Energy Rev., 54, pp. 1212-1239.

[24] Garoosi, F., Bagheri, G., and Rashidi, M. M., 2015. "Two phase simulation of natural convection and mixed convection of the nanofluid in a square cavity". Powder Technol., 275, pp. 239-256.

[25] Haddad, Z., Abu-Nada, E., Oztop, H. F., and Mataoui, A., 2012. "Natural convection in nanofluids: are the thermophoresis and Brownian motion effects significant in nanofluid heat transfer enhancement?'. Int. J. Therm. Sci., 57, pp. 152-162.

[26] Dastmalchi, M., Sheikhzadeh, G. A., and Arani, A. A. A., 2015. "Double-diffusive natural convective in a porous square enclosure filled with nanofluid". Int. J. Therm. Sci., 95, pp. 88-98.

[27] Siddheshwar, P. G., and Kanchana, C., 2017. "Unicellular unsteady Rayleigh-Bénard convection in Newtonian liquids and Newtonian nanoliquids occupying enclosures: New findings". Int. J. Mech. Sci., 131, pp. 1061-1072.

[28] Siddheshwar, P. G., and Kanchana, C., 2018. "A study of unsteady, unicellular Rayleigh-Bénard convection of nanoliquids in enclosures using additional modes". $J$. Nanofluids, 7, pp. 791-800.

[29] Kanchana, C., and Zhao, Y., 2018. "Effect of internal heat generation/absorption on Rayleigh-Bénard convection in water well-dispersed with nanoparticles on carbon nanotubes". Int. J. Heat Mass Transfer, 127, pp. 1031-1047.

[30] Kanchana, C., Zhao, Y., and Siddheshwar, P. G., 2018. "A comparative study of individual influences of suspended multiwalled carbon nanotubes and alumina nanoparticles on Rayleigh-Bénard convection in water". Phys. Fluids, 30, pp. 084101-1-084101-14.

[31] Park, H. M., 2015. "Rayleigh-Bénard convection of nanofluids based on the pseudo-single-phase continuum model". Int. J. Therm. Sci., 90, pp. 267-278.

[32] Corcione, M., 2011. "Rayleigh-Bénard convection heat transfer in nanoparticle suspensions". Int. J. Heat Fluid Flow, 32, pp. 65-77.

[33] D’Orazio, M. C., Cianfrini, C., and Corcione, M., 2004. "Rayleigh-Bénard convection in tall rectangular enclosures". Int. J. Therm. Sci., 43, pp. 135-144.

[34] Abu-Nada, E., and Chamkha, A. J., 2010. "Effect of nanofluid variable properties on natural convection in enclosures filled with a Cuo-EG-water nanofluid". Int. J. Therm. Sci., 49, pp. 2339-2352.

[35] Jou, R.-Y., and Tzeng, S.-C., 2006. "Numerical research of nature convective heat transfer enhancement filled with nanofluids in rectangular enclosures". Int. Comm. Heat Mass Transfer, 33, pp. 727-736.

[36] Elhajjar, B., Bachir, G., Mojtabi, A., Fakih, C., and Charrier-Mojtabi, M. C., 2010. "Modeling of Rayleigh-Bénard natural convection heat transfer in nanofluids". C. R. Mécanique, 338, pp. 350-354.

[37] Abu-Nada, E., Masoud, Z., Oztop, H. F., and Campo, A., 2010. "Effect of nanofluid variable properties on natural convection in enclosures". Int. J. Therm. Sci., 49, pp. 479-491.

[38] Groşan, T., Revnic, C., Pop, I., and Ingham, D. B., 2015. "Free convection heat transfer in a square cavity filled with a porous medium saturated by a nanofluid". Int. J. Heat Mass Transfer, 87, pp. 36-41.

[39] Sheremet, M. A., Pop, I., and Nazar, R., 2015. "Natural convection in a square cavity filled with a porous medium saturated with a nanofluid using the thermal nonequilibrium model with a Tiwari and Das nanofluid model". Int. J. Mech. Sci., 100, pp. 312-321.

[40] Sheremet, M. A., Pop, I., and Rahman, M. M., 2015. "Three-dimensional natural convection in a porous enclosure filled with a nanofluid using Buongiorno's mathematical model". Int. J. Heat Mass Transfer, 82, pp. 396-405.

[41] Brinkman, H. C., 1952. "The viscosity of concentrated suspensions and solutions”. J. Chem. Phy., 20, pp. 571.

[42] Bianco, V., Manca, O., Nardini, S., and Vafai, K., 2015. Heat transfer enhancement with nanofluids. CRC Press, New York, USA.

[43] Hamilton, R. L., and Crosser, O. K., 1962. "Thermal conductivity of heterogeneous two-component systems". Ind. Eng. Chem. Fundam., 1, pp. 187-191.

[44] Koo, J., and Kleinstreuer, C., 2004. "A new thermal conductivity model for nanofluids". J. Nanopart. Res., 6(6), pp. 577-588.

[45] Chandrasekhar, S., 1961. Hydrodynamic and hydromagnetic stability. Clarendon Press, London.

[46] Chandrasekhar, S., and Reid, W. H., 1957. "On the expansion of functions which satisfy four boundary conditions". Proceedings of the National Academy of Sciences of the United States of America, 43, pp. 521-527.

[47] Nagata, M., 1995. "Bifurcations at the Eckhaus points in two-dimensional Rayleigh-Bénard convection". Phys. Rev. E, 52, pp. 6141-6145.

[48] Oztop, H. F., and Abu-Nada, E., 2008. "Numerical study of natural convection in partially heated rectangular enclosures filled with nanofluids". Int. J. Heat Fluid Flow, 29, pp. 1326-1336.

[49] "https://www.matbase.com

[50] Baines, P. G., and Gill, A. E., 1969. "On thermohaline convection with linear gradients". J. Fluid Mech., 37, pp. 289-306. 